\title{
Influence of sulfite and fluoride soil contamination on the pigment content in some species of arboreal plants
}

\author{
Yuriy Prysedsky ${ }^{1 *}$ \\ Yuriy Lykholat ${ }^{2}$ \\ ${ }^{1}$ Vasyl Stus Donetsk \\ National University, \\ 21 600-richchya, \\ Vinnytsia 21021, Ukraine \\ ${ }^{2}$ Oles Honchar Dnipropetrovsk \\ National University, \\ 72 Haharina Ave., \\ Dnipro 49000, Ukraine
}

Industrial pollution of soil exerts a great negative influence on plant organisms. In particular, plants that grow in soil polluted with high-density metals, sulfur compounds $\left(\mathrm{SO}_{4}^{2-}\right)$, chlorine $\left(\mathrm{Cl}^{-}\right)$, etc., are characterized by growth inhibition and reduced metabolic activity. Additionally, plants play a significant role in optimizing the environment.

The objects of the investigation were sprouts of five woody plant species - Gleditcia triacantos L., Quercus robur L., Lonicera tataricum L., Eleagnus angustifolia L., Robinia pseudoacacia L., which were cultivated in the soil contaminated with fluoride and sulfite

The content of chlorophyll $a$ in the stable plants (Eleagnus angustifolia L. and Robinia pseudoacacia L.) decreases by 6.2-190.0\%, and chlorophyll $b$ decreases by $3.1-124.6 \%$ in comparison to control plants. All variations of pollutants inserted into soil cause a significant decrease in the level of chlorophyll $a$ (14.7-55.4\%) for not-stable Lonicera tataricum $\mathrm{L}$. The amount of chlorophyll $b$ decreases 1.45-1.88 times for this species.

For stable species Eleagnus angustifolia L. and Robinia pseudoacacia $\mathrm{L}$., the changes in the the content of carotenoids are characterized by their 4.5-5.5-times decrease in comparison to the control plants. For unstable species (Gleditcia triacantos L., Lonicera tataricum L.), the content of carotenoids decreases by $36.5-86.3 \%$.

Keywords: fluoride, sulphite, soil pollution, woods and bushes plants, photosynthetic pigments

\section{INTRODUCTION}

The existing state of rising demand requires significant manufacturing development that is accompanied by considerable pervasion of substances extrinsic to nature (Varshney, Garg, 1980; Ilkun, 1978; El-Shazly et al., 1990). The largest

\footnotetext{
*Corresponding author. Email: yu.prysedskyi@donnu.edu.ua
}

part of pollutants comes into the atmosphere as gaseous substances and aerosols. Under the influence of ultraviolet radiation, oxygen, and other factors, gases transform into aerosols of acids and salt aerosols (Ilkun, 1978; Agrawal et al., 1985). Liquid and solid aerosols accumulate in the soil and cause its contamination.

Industrial pollution of soil has a great negative impact on plants. In particular, plants that grow in soil polluted with high-density metals, sulfur 
compounds $\left(\mathrm{SO}_{4}^{2-}\right)$, chlorine $\left(\mathrm{Cl}^{-}\right)$, etc., are characterized by growth inhibition and reduced metabolic activity (Negrutskaja et al., 2005; Domingos et al., 2003; Bobrova et al., 2010). As a rule, these plants are characterized by depressed photosynthesis (Ebregt et al., 1977; Lykholat et al., 2016; Prysedskyj, 2014), a reduced content of photosynthetic pigments, and damaged chlorophyll fluorescence (Pukacki, 2000; Matysiak, 2001). Furthermore, plants are significantly important for environment optimizing when it concerns anthropogenically transformed medium. Consequently, the aim of our research was to investigate the changes in the content of photosynthetic pigments in woody plants when soil is contaminated with fluorides and sulphites.

\section{MATERIALS AND METHODS}

\section{Plant material}

The objects for the investigation were 30 -dayold sprouts of five woody plant species such as Gleditcia triacantos L., Quercus robur L., Lonicera tataricum L., Eleagnus angustifolia L., Robinia pseudoacacia L., which were grown in soil contaminated with fluorides and sodium sulfite.

\section{Efflux experiments}

Research was conducted according to the plan for a complete two-coefficient three-tier experiment (Table 1).

The spectrophotometric method was applied in the investigation to define the content of photosynthetic pigments in the acetone extract. The amount of pigments was calculated according to Vetshtein's formulas (Prysedsky, 2016).

\section{Statistical analysis}

All experiments were conducted iteratively - six times (sample size). The results were statistically processed according to the scheme of two-factors dispersion nalysis. The Dunnett's method was leading in comparison of the mean values (Prysedsky, 1999; Prysedsky, 2005). The influence of factors was believed to be valid if the calculated value of the Fisher criteria exceeded the standard $\alpha=0.05$. The differ- ence between the average values was considered valid if the absolute value of the difference between the control and experimental values (D) exceeded the Dannet deviation $\left(\mathrm{D}^{\mathrm{D}}\right)$ (the least meaningful difference) $\alpha=0.05$.

\section{RESULTS AND DISCUSSION}

The results of previous researches show that the species used for pigment group study could be ranged by the degree of growth depression in the following way: Eleagnus angustifolia L., Robinia pseudoacacia L., Quercus robur L., Gleditcia triacantos L., Lonicera tataricum L. Moreover, it was determined that Quercus robur L. was greatly susceptible to the pollution with sulfites.

Chlorophylls are the main photosynthetic pigments involved in the absorbtion of light energy in plants. The results of the investigation show the variability of the reaction of experimental species to the content of chlorophylls $a$ and $b$ in polluted soil (Table 2 ). So, depending on the composition and on the content of the pollutants in the soil the content of chlorophyll $a$ decreases by $6.2-190.0 \%$, and that of chlorophyll $b$ by 3.1-124.6 \% for stable species Eleagnus angustifolia L. and Robinia pseudoacacia L. in comparison to the plants which grow in unpolluted soil.

For Quercus robur L., the content of chlorophyll did not change significantly for the most variants used in the research, although sulfite and complex pollution tended to decrease the amount of pigments. For not stable Lonicera tataricum L., all the variants of inserting the pollutants into soil caused considerable (14.7-55.4\%) reduction of chlorophyll $a$. The amount of chlorophyll $b$ also decreased 1.45-1.88 times for the sprouts of these species in comparison to the control plants.

The analogous outcome was observed in the changes in the total amount of green pigments; nevertheless, these changes are feebly marked.

Carotenoids played a significant role both in the processes of photosynthesis and in the elimination of oxidative stress of plants.

Analysis of the data in Table 3 shows that the content of carotenoids greatly increased under the influence of all combinations of 
Table 1. Plan of the experiment

\begin{tabular}{|c|c|c|c|c|c|c|c|c|}
\hline \multirow{2}{*}{ 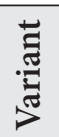 } & \multicolumn{2}{|c|}{ Content of pollutants } & \multirow{2}{*}{ 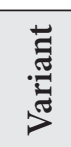 } & \multicolumn{2}{|c|}{ Content of pollutants } & \multirow{2}{*}{ 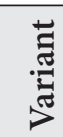 } & \multicolumn{2}{|c|}{ Content of pollutants } \\
\hline & $\mathrm{F}^{-}, \mathrm{mg} / \mathrm{kg}$ & $\mathrm{S}^{2-}, \mathrm{g} / \mathrm{kg}$ & & $\mathrm{F}^{-}, \mathrm{mg} / \mathrm{kg}$ & $\mathrm{S}^{2-}, \mathrm{g} / \mathrm{kg}$ & & $\mathrm{F}^{-}, \mathrm{mg} / \mathrm{kg}$ & $\mathrm{S}^{2-}, \mathrm{g} / \mathrm{kg}$ \\
\hline 1 & 0 & 0 & 4 & 0 & 1 & 7 & 0 & 2 \\
\hline 2 & 100 & 0 & 5 & 100 & 1 & 8 & 100 & 2 \\
\hline 3 & 200 & 0 & 6 & 200 & 1 & 9 & 200 & 2 \\
\hline
\end{tabular}

Table 2. Influence of soil pollution on the chlorophylls content of woody and bushy plants

\begin{tabular}{|c|c|c|c|c|c|c|c|c|}
\hline \multirow{3}{*}{ 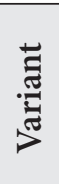 } & \multicolumn{8}{|c|}{ Chlorophyll content, $\mathrm{mg} / \mathrm{g}$} \\
\hline & \multicolumn{4}{|c|}{ Chlorophyll a } & \multicolumn{4}{|c|}{ Chlorophyll b } \\
\hline & $\mathbf{M} \pm \mathbf{m}$ & $\mathrm{D}$ & DD & $\%$ for control & $\mathrm{M} \pm \mathrm{m}$ & $\mathrm{D}$ & $\mathrm{DD}$ & $\%$ for control \\
\hline \multicolumn{9}{|c|}{ Gleditchia triacantos $\mathrm{L}$. } \\
\hline 1 & $0.25 \pm 0.05$ & & & 100.0 & $0.69 \pm 0.13$ & & & 100.0 \\
\hline 2 & $0.34 \pm 0.05$ & 0.09 & 0.80 & 136.0 & $0.92 \pm 0.14$ & 0.22 & 4.07 & 133.3 \\
\hline 3 & $0.91 \pm 0.61$ & 0.66 & 0.80 & 364.0 & $0.71 \pm 0.19$ & 0.02 & 4.07 & 102.9 \\
\hline 4 & $0.53 \pm 0.16$ & 0.28 & 0.80 & 112.0 & $1.26 \pm 0.30$ & 0.57 & 4.07 & 182.6 \\
\hline 5 & $0.68 \pm 0.26$ & 0.42 & 0.80 & 272.0 & $0.67 \pm 0.11$ & -0.03 & 4.07 & 97.1 \\
\hline 6 & $0.35 \pm 0.18$ & 0.10 & 0.80 & 140.0 & $5.11 \pm 3.33$ & 4.41 & 4.07 & 740.5 \\
\hline 7 & $0.33 \pm 0.05$ & 0.08 & 0.80 & 132.0 & $1.21 \pm 0.30$ & 0.52 & 4.07 & 175.3 \\
\hline 8 & $0.50 \pm 0.10$ & 0.24 & 0.80 & 200.0 & $0.93 \pm 0.18$ & 0.23 & 4.07 & 134.8 \\
\hline 9 & $0.70 \pm 0.22$ & 0.45 & 0.80 & 280.0 & $1.55 \pm 0.54$ & 0.85 & 4.07 & 224.6 \\
\hline \multicolumn{9}{|c|}{ Quercus robur $\mathrm{L}$. } \\
\hline 1 & $0.08 \pm 0.03$ & & & 100.0 & $0.26 \pm 0.03$ & & & 100.0 \\
\hline 2 & $0.06 \pm 0.01$ & 0.03 & 0.25 & 67.1 & $0.23 \pm 0.05$ & 0.03 & 0.36 & 88.5 \\
\hline 3 & $0.05 \pm 0.01$ & 0.03 & 0.25 & 63.4 & $0.21 \pm 0.04$ & 0.05 & 0.36 & 80.8 \\
\hline 4 & $0.24 \pm 0.14$ & 0.16 & 0.25 & 295.1 & $0.25 \pm 0.05$ & 0.01 & 0.36 & 95.0 \\
\hline 5 & $0.08 \pm 0.02$ & 0.01 & 0.25 & 91.5 & $0.12 \pm 0.03$ & 0.14 & 0.36 & 49.1 \\
\hline 6 & $0.07 \pm 0.03$ & 0.01 & 0.25 & 83.4 & $0.11 \pm 0.04$ & 0.15 & 0.36 & 43.5 \\
\hline 7 & $0.09 \pm 0.03$ & 0.01 & 0.25 & 110.9 & $0.37 \pm 0.15$ & 0.11 & 0.36 & 141.1 \\
\hline 8 & $0.08 \pm 0.02$ & 0.00 & 0.25 & 97.6 & $0.15 \pm 0.02$ & 0.11 & 0.36 & 58.8 \\
\hline 9 & $0.32 \pm 0.14$ & 0.24 & 0.25 & 390.2 & $0.54 \pm 0.24$ & 0.28 & 0.36 & 208.8 \\
\hline \multicolumn{9}{|c|}{ Lonicera tatarica $\mathrm{L}$. } \\
\hline 1 & $0.54 \pm 0.10$ & & & 100.0 & $3.05 \pm 1.74$ & & & 100.0 \\
\hline 2 & $0.41 \pm 0.04$ & 0.13 & 0.67 & 76.1 & $1.62 \pm 0.14$ & 0.43 & 2.27 & 53.1 \\
\hline 3 & $0.91 \pm 0.53$ & 0.36 & 0.67 & 166.7 & $1.84 \pm 0.63$ & 1.21 & 2.27 & 60.3 \\
\hline 4 & $0.40 \pm 0.04$ & 0.15 & 0.67 & 72.7 & $1.64 \pm 0.15$ & 1.41 & 2.27 & 53.8 \\
\hline 5 & $0.24 \pm 0.06$ & 0.30 & 0.67 & 44.6 & $1.99 \pm 0.48$ & 1.06 & 2.27 & 65.2 \\
\hline 6 & $0.30 \pm 0.07$ & 0.25 & 0.67 & 54.7 & $1.95 \pm 0.50$ & 1.10 & 2.27 & 63.9 \\
\hline 7 & $0.25 \pm 0.07$ & 0.30 & 0.67 & 45.5 & $1.95 \pm 0.56$ & 1.10 & 2.27 & 63.9 \\
\hline 8 & $0.46 \pm 0.04$ & 0.08 & 0.67 & 85.3 & $1.96 \pm 0.26$ & 0.09 & 2.27 & 64.3 \\
\hline 9 & $0.34 \pm 0.07$ & 0.20 & 0.67 & 62.9 & $2.11 \pm 0.49$ & 0.94 & 2.27 & 69.2 \\
\hline
\end{tabular}




\begin{tabular}{|c|c|c|c|c|c|c|c|c|}
\hline \multirow{3}{*}{ 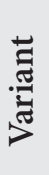 } & \multicolumn{8}{|c|}{ Chlorophyll content, $\mathrm{mg} / \mathrm{g}$} \\
\hline & \multicolumn{4}{|c|}{ Chlorophyll a } & \multicolumn{4}{|c|}{ Chlorophyll b } \\
\hline & $\mathrm{M} \pm \mathrm{m}$ & $\mathrm{D}$ & $\mathrm{DD}$ & $\%$ for control & $M \pm m$ & $\mathrm{D}$ & $\mathrm{DD}$ & $\%$ for control \\
\hline \multicolumn{9}{|c|}{ Elesgnus angustifolia $\mathrm{L}$. } \\
\hline 1 & $0.31 \pm 0.02$ & & & 100.0 & $0.15 \pm 0.01$ & & & 100.0 \\
\hline 2 & $0.71 \pm 0.15$ & 0.41 & 0.73 & 229.0 & $2.35 \pm 2.40$ & 2.20 & 3.18 & 1566.7 \\
\hline 3 & $0.58 \pm 0.22$ & 0.28 & 0.73 & 187.1 & $2.0 \pm 1.3$ & 1.95 & 3.18 & 1400.0 \\
\hline 4 & $0.39 \pm 0.08$ & 0.09 & 0.73 & 125.8 & $0.27 \pm 0.04$ & 0.12 & 3.18 & 180.0 \\
\hline 5 & $0.67 \pm 0.28$ & 0.36 & 0.73 & 216.1 & $0.34 \pm 0.10$ & 0.19 & 3.18 & 226.7 \\
\hline 6 & $0.22 \pm 0.02$ & 0.09 & 0.73 & 70.9 & $0.70 \pm 0.1$ & 0.55 & 3.18 & 466.7 \\
\hline 7 & $0.14 \pm 0.03$ & 0.17 & 0.73 & 45.2 & $0.22 \pm 0.15$ & 0.07 & 3.18 & 146.7 \\
\hline 8 & $0.40 \pm 0.05$ & 0.09 & 0.73 & 129.0 & $0.89 \pm 0.12$ & 0.74 & 3.18 & 593.3 \\
\hline 9 & $0.21 \pm 0.04$ & 0.21 & 0.73 & 67.7 & $0.08 \pm 0.01$ & -0.07 & 3.18 & 53.3 \\
\hline \multicolumn{9}{|c|}{ Robinia pseudoacacia L. } \\
\hline 1 & $0.48 \pm 0.05$ & & & 100.0 & $0.32 \pm 0.02$ & & & 100.0 \\
\hline 2 & $0.57 \pm 0.05$ & 0.09 & 0.23 & 118.7 & $0.39 \pm 0.04$ & 0.06 & 0.14 & 121.9 \\
\hline 3 & $0.51 \pm 0.11$ & 0.03 & 0.23 & 106.2 & $0.33 \pm 0.05$ & 0.01 & 0.14 & 103.1 \\
\hline 4 & $0.52 \pm 0.04$ & 0.04 & 0.23 & 108.3 & $0.39 \pm 0.05$ & 0.07 & 0.14 & 121.9 \\
\hline 5 & $0.64 \pm 0.09$ & 0.16 & 0.23 & 133.3 & $0.36 \pm 0.03$ & 0.04 & 0.14 & 112.5 \\
\hline 6 & $0.39 \pm 0.05$ & 0.09 & 0.23 & 81.2 & $0.22 \pm 0.03$ & 0.10 & 0.14 & 68.7 \\
\hline 7 & $0.44 \pm 0.06$ & 0.05 & 0.23 & 91.7 & $0.33 \pm 0.06$ & 0.01 & 0.14 & 103.1 \\
\hline 8 & $0.55 \pm 0.06$ & 0.07 & 0.23 & 114.6 & $0.31 \pm 0.04$ & 0.01 & 0.14 & 96.9 \\
\hline 9 & $0.42 \pm 0.05$ & 0.06 & 0.23 & 87.5 & $0.25 \pm 0.02$ & 0.07 & 0.14 & 72.0 \\
\hline
\end{tabular}

Table 3. Influence of soil pollution on the content of carotenoids in woody and bushy plants

\begin{tabular}{|c|c|c|c|c|}
\hline \multirow{2}{*}{$\begin{array}{c}\text { Vari- } \\
\text { ant }\end{array}$} & $\mathbf{4} \pm \mathbf{m}$ & $\mathrm{D}$ & $\mathrm{D}^{\mathrm{D}}$ & $\begin{array}{c}\text { Carotenoids con- } \\
\text { trol }\end{array}$ \\
\cline { 2 - 5 } & \multicolumn{5}{|c|}{ Gleditchia triacantos L. } \\
\hline 1 & $0.36 \pm 0.24$ & & & 100.0 \\
\hline 2 & $0.10 \pm 0.03$ & -0.26 & 1.14 & 27.6 \\
\hline 3 & $0.21 \pm 0.0$ & -0.15 & 1.14 & 58.0 \\
\hline 4 & $0.22 \pm 0.02$ & -0.15 & 1.14 & 59.9 \\
\hline 5 & $0.12 \pm 0.05$ & -0.25 & 1.14 & 32.3 \\
\hline 6 & $1.12 \pm 0.91$ & 0.76 & 1.14 & 310.2 \\
\hline 7 & $0.14 \pm 0.04$ & -0.22 & 1.14 & 39.2 \\
\hline 8 & $0.19 \pm 0.04$ & -0.17 & 1.14 & 51.9 \\
\hline 9 & $0.16 \pm 0.03$ & -0.20 & 1.14 & 43.7 \\
\hline \multicolumn{5}{|c|}{ Quercus robur L. } \\
\hline 1 & $0.02 \pm 0.01$ & & & 100.0 \\
\hline
\end{tabular}

the pollutants for stable species Eleagnus angustifolia L. and Robinia pseudoacacia L. In particular, a considerable increase of this indicator became apparent for Robinia pseudoacacia L. Thus the number of the pigments increased 4.5-5.5 times for variants that involved fluoride and complex contamination in comparison to the plants that grew in unpolluted soil.

Depending on the composition and dosage of pollutants, the content of carotenoids decreased by $36.5-86.3 \%$ for unstable species (Gleditcia triacantos L., Lonicera tataricum L.). For Quercus robur L. fluoride pollution causes decrease of carotenoid content by $66.7-577.8 \%$, and sulfide contamination causes reduction of the amount of these pigments.

The results of the investigation conform with other research on the impact of environment pollution on the pigment content in plants. Also they 
Table 3 (continued)

\begin{tabular}{|c|c|c|c|c|}
\hline \multirow{2}{*}{$\begin{array}{c}\text { Vari- } \\
\text { ant }\end{array}$} & \multicolumn{4}{|c|}{ Carotenoids content, mg/g } \\
\cline { 2 - 5 } & $\mathbf{M} \pm \mathbf{m}$ & $\mathrm{D}$ & $\mathrm{D}^{\mathrm{D}}$ & $\begin{array}{c}\% \text { for con- } \\
\text { trol }\end{array}$ \\
\hline 2 & $0.02 \pm 0.01$ & -0.001 & 0.35 & 94.4 \\
\hline 3 & $0.03 \pm 0.01$ & 0.01 & 0.35 & 166.7 \\
\hline 4 & $0.05 \pm 0.02$ & 0.03 & 0.35 & 250.0 \\
\hline 5 & $0.07 \pm 0.03$ & 0.05 & 0.35 & 372.2 \\
\hline 6 & $0.08 \pm 0.02$ & 0.06 & 0.35 & 444.4 \\
\hline 7 & $0.11 \pm 0.03$ & 0.09 & 0.35 & 583.3 \\
\hline 8 & $0.12 \pm 0.03$ & 0.10 & 0.35 & 677.8 \\
\hline 9 & $0.51 \pm 0.29$ & 0.49 & 0.35 & 2844.4 \\
\hline
\end{tabular}

Lonicera tatarica $\mathrm{L}$.

\begin{tabular}{|c|c|c|c|c|}
\hline 1 & $0.82 \pm 0.49$ & & & 100.0 \\
\hline 2 & $0.60 \pm 0.40$ & -0.22 & 0.85 & 72.8 \\
\hline 3 & $0.3 \pm 0.03$ & -0.70 & 0.85 & 15.2 \\
\hline 4 & $0.12 \pm 0.05$ & -0.70 & 0.85 & 14.6 \\
\hline 5 & $0.15 \pm 0.04$ & -0.67 & 0.85 & 18.7 \\
\hline 6 & $0.09 \pm 0.03$ & -0.73 & 0.85 & 10.6 \\
\hline 7 & $0.17 \pm 0.14$ & -0.65 & 0.85 & 20.98 \\
\hline 8 & $0.55 \pm 0.46$ & -0.28 & 0.85 & 66.5 \\
\hline 9 & $0.11 \pm 0.03$ & -0.71 & 0.85 & 13.7 \\
\hline
\end{tabular}

Elesgnus angustifolia $\mathrm{L}$.

\begin{tabular}{|c|c|c|c|c|}
\hline 1 & $0.09 \pm 0.02$ & & & 100.0 \\
\hline 2 & $0.11 \pm 0.01$ & 0.01 & 0.11 & 115.1 \\
\hline 3 & $0.07 \pm 0.01$ & -0.02 & 0.11 & 77.4 \\
\hline 4 & $0.10 \pm 0.01$ & 0.002 & 0.11 & 102.2 \\
\hline 5 & $0.11 \pm 0.02$ & 0.02 & 0.11 & 118.3 \\
\hline 6 & $0.11 \pm 0.02$ & 0.02 & 0.11 & 121.5 \\
\hline 7 & $0.12 \pm 0.03$ & 0.02 & 0.11 & 123.7 \\
\hline 8 & $0.07 \pm 0.03$ & -0.02 & 0.11 & 78.5 \\
\hline 9 & $0.32 \pm 0.07$ & 0.22 & 0.11 & 338.7 \\
\hline \multicolumn{5}{|c|}{ Robinia pseudoacacia L. } \\
\hline 1 & $0.06 \pm 0.01$ & & & 100.0 \\
\hline 2 & $0.27 \pm 0.03$ & 0.21 & 0.15 & 450.0 \\
\hline 3 & $0.33 \pm 0.07$ & 0.27 & 0.15 & 550.0 \\
\hline 4 & $0.24 \pm 0.05$ & 0.18 & 0.15 & 400.0 \\
\hline 5 & $0.22 \pm 0.04$ & 0.16 & 0.15 & 366.7 \\
\hline 6 & $0.27 \pm 0.04$ & 0.21 & 0.15 & 450.0 \\
\hline 7 & $0.21 \pm 0.02$ & 0.15 & 0.15 & 350.0 \\
\hline 8 & $0.21 \pm 0.05$ & 0.15 & 0.15 & 350.0 \\
\hline 9 & $0.32 \pm 0.07$ & 0.26 & 0.15 & 533.3 \\
\hline
\end{tabular}

allow using the analysis of the pigment complex of the plants for the diagnosis of the state of plants under the conditions of soil contamination.

\section{CONCLUSIONS}

Research on the influence of soil polluted with fluorides and sulphides on the content of photosynthetic pigments in the woody sprouts and shrubs shows that stable species Eleagnus angustifolia L. and Robinia pseudoacacia L. are characterized by an increased content of green pigments and carotenoids under the conditions of soil contamination. Such a reaction might play a significant role in the adaptation of stable plants to pollutants impact.

Unstable Lonicera tataricum L. is susceptible to contamination soil pigment complex that reduces the adaptive capacity of such plant species.

Received 18 July 2017 Accepted 12 September 2017

\section{References}

1. Ebregt JM, Boldewijn A, Ebregt JM, Boldewij AM. Influence of heavy metals in spruce forest soil on amylase activity, $\mathrm{CO}_{2}$ evolution from starch and soil respiration. Plant and Soil. 1977; 47(1): 137-48.

2. Varshney K, Garg KK. Significance of leaf surface characteristics in plant responses to air pollution. Water, Air, and Soil Pollution. 1980; 14(1): 429-33.

3. Ilkun GM. Zagryazniteli atmosfery i rasteniya. Kiev: Naukova dumka, 1978. 247 p. Russian.

4. Negrutskaya GM, Prisedskiy YuG, Zyuzyukina LV, Pryidyibaylo SV. Izmenenie rostovyih protsessov $\mathrm{v}$ prorostkah drevesnyih rasteniy pri sulfyitnom i ftoridnom zagryaznenii pochvyi. Introduktsiya i akklimatizatsiya rasteniy. 2005; 24: 45-52. Russian.

5. Agrawal M, Nandi PK, Rao DN. Effects of sulphur dioxide fumigation on soil system and growth behaviour of Vicia faba plants. Plant and Soil.1985; 86(1): 69-78. 
6. Domingos M, Rinaldi MCS, Modesto IF, Klumpp G, Delitti WBC, Domingos M. Combined effects of air and soil pollution by fluoride emissions on Tibouchina pulchra Cogn., at Cubatão, SE Brazil, and their relations with aluminium. Plant and Soil. 2003; 249(2): 297-308.

7. Bobrova OM, Liholat YuV, Grigoryuk IP, Serga AI, Yavorovskiy PP. Aktivnist antioksidantnih fermentiv u listi riznih vidiv barbarisu (Berberis L.) za diyi vazhkih metaliv. Naukovi dopovidi NUBIP. 2010; 5(10): 1-10. Ukrainian.

8. Pukacki PM. Effects of sulphur, fluoride and heavy metal pollution on the chlorophyll fluorescence of Scots pine (Pinus sylvestris L.) needles. Dendrobiology. 2000; 45: 83-8.

9. Matysiak R. Content of carotenoids in needles of Pinus sylvestris L. growing in a polluted area. Dendrobiology. 2001; 46: 39-42.

10. El-Shazly SM, Abdelmageed AM, Abdelaal A. Studies on pollution in the atmosphere near the aluminium reduction plant at Nagi Hammady. Egypt Water, Air, and Soil Pollution. 1990; 51(3): 217-30.

11. Lykholat Y, Alekseeva A, Khromykh N, Ivan'ko I, Kharytonov M, Kovalenko I. Assessment and prediction of viability and metabolic activity of Tilia platyphyllos in arid steppe climate of Ukraine. Agriculture \& Forestry. 2016; 62(3): 57-64.

12. Prisedskiy YuG. Vpliv zabrudnennya gruntu ftori dami ta sulfitami na rostovi pokazniki deyakih vidiv kvit-kovo-dekorativnih roslin. Visnik Dnipropetrovskogo derzhavnogo agrarno-ekonomichnogo universitetu. 2014; 1: 115-9. Ukrainian.

13. Prisedskiy YuG. Statistichna obrobka rezultativ biologichnih eksperimentiv. Donetsk: Kassiopeya, 1999. 210 p. Ukrainian.

14. Prisedskiy YuG. Paket program dlya provedennya statistichnoy obrobki rezultativ biologichnih eksperimentiv. Donetsk: DonNU, 2005. 92 p.

15. Prisedskiy YuG. Fotosintez. Metodichniy posibnik z vikonannya laboratornih robit ta samostiynoyi roboti. Vinnitsya: DonNU, 2016. 76 p.

\section{Yuriy Prysedskyj, Yuriy Lykholat}

\section{DIRVOŽEMIO TARŠOS SULFITU IR FLUO- RIDU ITTAKA KAI KURIŲ ARBOREALINIŲ AUGALŲ RŪŠIŲ PIGMENTŲ KIEKIUI}

\section{Santrauka}

Augalai vaidina svarbų vaidmeni optimizuojant aplinką. Pramoninè dirvožemio tarša daro didelę neigiamą ittaką augalams. Visų pirma slopinamas augalų, augančių metalais, sieros junginiais $\left(\mathrm{SO}_{4}{ }^{2-}\right)$, chloru $\left(\mathrm{Cl}^{-}\right)$ir kitais junginiais užterštame dirvožemyje, augimas, sumažeja jų metabolinis aktyvumas. Šio tyrimo metu buvo tirtos penkios medžių rūšys: Gleditcia triacantos L., Quercus robur L., Lonicera tataricum L., Eleagnus angustifolia L., Robinia pseudoacacia L., augintos fluoridu ir sulfitu užterštame dirvožemyje. Palyginti su kontroliniais augalais, E. angustifolia ir R. pseudoacacia rūšies augaluose $a$ chlorofilo kiekis sumažèja 6,2-190,0 \%, o $b$ chlorofilo - 3,1-124,6 \%. Stabilių rūšių (E. angustifolia ir R. pseudoacacia) karotinoidų kiekis mažeja 4,5-5,5 karto, palyginti su kontroliniais augalais, nestabilių rūšių (G. triacantos ir L. tataricum) sumažèja 36,5-86,3 \%.

Raktažodžiai: fluoridas, sulfitas, dirvožemio tarša, medžiai, krūmai, fotosintetiniai pigmentai 